\title{
Perceived importance of being thin and smoking initiation among young girls
}

\section{K Honjo, M Siegel}

Tobacco Control 2003;12:289-295

Correspondence to: Kaori Honjo, MPH, Okayama University Graduate School of Medicine and Dentistry, Department of Hygiene and Preventive Medicine, 2-5-1 Shikata-cho, Okayama, Japan 700-8558.

khonjoJ@md.okayama-u.ac.jp

Received 10 March 2002. Accepted 9 April 2003
Background: Smoking among adolescents remains unacceptably high and the difference in potential risk factors for smoking initiation between male and female adolescents has been explored. Although the association between smoking initiation and dieting behaviour has been observed among girls, the mechanism of the association is unknown.

Objective: To examine prospectively the association between perceived importance of being thin at baseline and smoking initiation among girls.

Design: A four year prospective cohort survey including perceived importance of being thin at baseline and smoking behaviour, conducted in 1993 and 1996.

Setting and participants: 273 Massachusetts female adolescents aged 12-15 years at baseline who reported having smoked no more than one cigarette by the time of the baseline survey, drawn from households sampled by random digit dialling.

Main outcome measure: Progression to established smoking, defined as having smoked 100 or more cigarettes in their lifetime.

Results: After adjusting for age, smoking status at baseline, and race/ethnicity, girls who valued thinness most strongly and somewhat strongly were both more likely to have become established smokers, compared to the girls who valued thinness least strongly. The odds ratios are $4.5195 \%$ confidence interval (Cl), 1.4 to 16.7$)$ and $3.4(95 \% \mathrm{Cl} 1.04$ to 10.9), respectively.

Conclusions: The level of perceived importance of being thin among young female adolescents predicts future smoking initiation. Smoking prevention programmes designed for female adolescents may therefore benefit from the inclusion of content related to importance of being thin.
D espite extensive knowledge of the health risks of smoking for several decades, adolescent smoking has continued to be a significant public health problem..$^{1-3}$ Considering the widespread prevalence of both weight concerns and smoking behaviour, especially among female adolescents, weight concerns have been hypothesised as one of the initiating factors for female adolescent smoking..$^{4-10}$ Although the relation between cigarette smoking and body weight has been established, the influence of weight concerns on smoking initiation is not clear. ${ }^{511}$

Over the past few decades, a great number of cross-sectional studies have been conducted on the relation between weight concerns and smoking behaviour. These studies have established a positive association between weight concerns and cigarette smoking among female adolescents but not among male adolescents. ${ }^{12-23}$ However, few prospective studies have been conducted to establish a causal relation between weight concerns and tobacco use. ${ }^{4511}$ French et al reported that girls who have dieted or constantly thought about weight at baseline were more likely to have initiated smoking one year later, compared to girls who do not have weight concerns. However, dieting and constant thoughts about weight did not predict smoking initiation among boys. ${ }^{12}$ Austin and Gortmaker have recently found that dieting frequency was prospectively associated with an increased risk of becoming a smoker among adolescent girls but not boys. ${ }^{24}$

The previously conducted studies on dieting and smoking suggest an association between weight concerns and smoking behaviour among female adolescents. ${ }^{11}{ }^{25}$ However, the temporal relation between weight concerns and smoking behaviour has not been clearly established and it is not entirely clear why smoking and weight concerns are associated.

The present study explored a possible underlying factor for the relation between weight concerns and smoking initiation.
We looked at "perceived importance of being thin" as a factor underlying adolescent girls' weight concerns. Perceived importance of being thin is defined as how much importance the respondent placed on being thin, which was measured by asking "On a scale of 0 to 10 , where 0 is not at all important and 10 is extremely important, how important is it to you to be slim or thin?".

The objective of this study was to examine whether the perceived importance of being thin is an initiating factor for smoking among younger female adolescents. Data come from a four year follow up telephone survey with a cohort of Massachusetts females, aged 12-15 years when surveyed in 1993. The survey included questions that allow us to examine the relation between perceived importance of being thin at baseline and subsequent rates of progression to established smoking, controlling for potential confounding factors, as described below.

This is the first study to examine whether the perceived importance of being thin is an initiating factor for smoking among younger female adolescents. The primary study hypotheses are: (1) young female adolescents who perceive that it is important to be thin at baseline are at higher risk of becoming smokers by follow up, compared to same age female adolescents who do not value thinness, controlling for possible confounding factors; and (2) the relation between the perceived importance of being thin at baseline and smoking initiation at follow up varies by the level of personal belief that smoking keeps weight down as measured at follow up.

Abbreviations: BMI, body mass index; OR, odds ratio; SES, socioeconomic status 


\section{METHOD}

\section{Design overview}

Data come from a four year follow up telephone survey with a cohort of Massachusetts females, aged 12-15 years when surveyed in 1993. The survey included questions that allow us to examine the relation between perceived importance of being thin at baseline and subsequent rates of progression to established smoking. We compared the rates of progression to established smoking across levels of perceived importance of thinness, using a multiple logistic regression model and controlling for potential confounding factors, as described below.

\section{Sample}

The 1993 Massachusetts Tobacco Survey, conducted by the Center for Survey Research, University of Massachusetts, Boston, was based on a probability sample of households drawn by use of random digit dialling. Based on brief screening interviews with adult informants in 11463 households, extended interviews were completed between October 1993 and March 1994 with 1069 youths aged 12-15 years, with a response rate of $75 \%$. Follow up interviews were conducted four years later. Thirty one per cent of the eligible youth $(\mathrm{n}=328)$ were not able to be tracked at follow up. Follow up interviews were completed with 618 of the remaining youth for an overall response rate of $57.8 \%$.

The sample for the present analysis was drawn from the 316 female subjects who were aged 12-15 years at baseline and who completed follow up interviews. We included only the 273 girls who reported having smoked no more than one cigarette by the time of the baseline survey.

\section{Measures}

Progression to established smoking

Following a classification scheme that was devised and validated by Pierce et $\mathrm{al}^{26}{ }^{27}$ we defined respondents who reported having smoked 100 or more cigarettes in their lifetime as established smokers. The theoretical rationale and validation of this measure of adolescent smoking have been established previously. ${ }^{26-29}$ This measure avoids the problem of the irregularity of smoking behaviour among adolescents and avoids using the unreliable recall of smoking behaviour in the last month to define established smoking behaviour. Self reports of smoking behaviour were not validated, because the survey was conducted by telephone.

\section{Perceived importance of being thin}

The predictor variable of this study is the perceived importance of being thin at baseline: how much importance the respondent placed on being thin. The perceived importance of being thin was measured by the following question: "On a scale of 0 to 10 , where 0 is not at all important and 10 is extremely important, how important is it to you to be slim or thin?" We categorised it into three levels, low (0-4), medium (5-7), and high (8-10). The proportions of subjects categorised into low, medium, and high in the study population are $15.7 \%, 40.8 \%$, and $43.4 \%$, respectively.

\section{Potential confounding variables}

To rule out the possibility that other factors confound the relation between perceived importance of being thin and progression to established smoking, we examined the effects of several potential confounding variables. These variables included (1) age, (2) race/ethnicity (non-Hispanic white $v$ other), (3) socioeconomic status (SES), (4) body mass index (BMI), (5) depression, and (6) baseline smoking status. All of these variables were measured at baseline. Based on prior research, ${ }^{3} 8^{25-27}$ 30-33 $^{-1}$ we expected age, race/ethnicity, SES, BMI, and depression to be associated with both the primary predictor (perceived importance of being thin) and the outcome (progression to established smoking). Because we hypothesised that perceived importance of being thin theoretically underlies dieting behaviour and an individual's evaluation of her own weight, we consider these to be mediating factors rather than confounding factors. Therefore, we did not control for them.

Interviewers asked subjects about their age, race/ethnicity, height, and weight, and obtained SES information from adult informants. BMI was calculated as self reported weight divided by self reported height squared $\left(\mathrm{kg} / \mathrm{m}^{2}\right)$. We classified subjects into three categories: $\mathrm{BMI}<20,20 \leqslant \mathrm{BMI}<23$, and $\mathrm{BMI} \geqslant 23$ in order to avoid the assumption of linear effect of BMI. Our classification scheme of BMI was based on reference standard, which indicated 85th centile of distribution as cut off for child obesity. ${ }^{34}$ Then, we classified the rest of girls, who were categorised as being not obese, by mean BMI.

SES was measured at the household level, using the educational level of the adult informant. Depression was measured with six items adapted from the Center for Epidemiologic Studies Depression Scale. ${ }^{35}$ The items addressed how often in the past year the respondents felt hopeless, felt depressed, had trouble sleeping, and so on. Internal consistency reliability was found to be at a satisfactory level (Chronbach's $\alpha=0.71$ ). ${ }^{37}$

Baseline smoking status was classified into three categories: (1) non-susceptible non-smokers, (2) susceptible nonsmokers, and (3) experimenters. Non-smokers were defined as girls who reported never having smoked at all. Nonsmokers were classified into two categories based on their susceptibility to smoking. The girls were classified as non-susceptible non-smokers if they answered "no" to the question "Do you think that you will try a cigarette soon?" and "definitely not" to the questions "If one of your best friends were to offer you a cigarette, would you smoke it?" and "At any time during the next year do you think you will smoke a cigarette?". This measure has been validated and has been shown to reliably predict progression to established smoking in previous studies. ${ }^{26}{ }^{38-40}$ Experimenters were defined as girls who reported to have puffed on or smoked one whole cigarette.

\section{Modifying variable}

Belief at baseline that smoking helps weight reduction was considered as a possible modifying factor in this analysis. The relation between perceived importance of being thin at baseline and progression to established smoking at follow up was hypothesised to vary by the level of personal belief that smoking helps weight reduction, as reported at baseline. The personal belief that smoking keeps weight down was assessed by asking "Do you believe that smoking helps people keep their weight down?".

\section{Data analysis}

We examined the predictors of progression to established smoking at follow up, hypothesising that greater perceived importance of being thin at baseline would be associated with higher odds of becoming an established smoker by follow up. We estimated the odds of becoming an established smoker using logistic regression with the SAS statistical package. ${ }^{41}$ Our model building approach was based on our theory. We first tested a univariate model with only the primary predictor, perceived importance of being thin. Then, primary covariates were added to the model, regardless of their significance. The primary covariates based on theory are smoking status, race, and age at baseline. The resulting model was considered our base model. Potential confounding variables (SES, BMI, and depression) were then added to the base model once at a time and then in combination. We kept covariates with a probability value of $\mathrm{p} \leqslant 0.20$ through the model building process until the best model was achieved. The standard errors for odds ratios were calculated using the Wald test. ${ }^{42}$ In order to test our second hypothesis, we examined the interactions between 


\begin{tabular}{|c|c|c|c|}
\hline \multirow[b]{2}{*}{ Characteristic } & \multirow[b]{2}{*}{$\%(n)$} & \multicolumn{2}{|c|}{$\begin{array}{l}\text { Perceived importance of being thir } \\
(0-10 \text { scale) }\end{array}$} \\
\hline & & Mean & $\mathrm{p}$ Value \\
\hline \multicolumn{4}{|l|}{ Age group } \\
\hline $12-13$ years & $54.8(149)$ & 6.56 & 0.547 \\
\hline $14-15$ years & 45.2 (123) & 6.78 & \\
\hline \multicolumn{4}{|l|}{ Race/ethnicity } \\
\hline White, non-Hispanic & $84.1(228)$ & 6.62 & 0.6905 \\
\hline Other & $15.9(43)$ & 6.82 & \\
\hline \multicolumn{4}{|l|}{ Education level of adult informant } \\
\hline High school or less & $44.4(120)$ & 6.74 & 0.6895 \\
\hline More than high school & $55.6(150)$ & 6.60 & \\
\hline \multicolumn{4}{|l|}{ Baseline smoking status $\dagger$} \\
\hline Non-susceptible non-smoker & $53.3(145)$ & 6.6 & 0.6733 \\
\hline Susceptible non-smoker & $22.4(61)$ & 6.48 & \\
\hline Smoked at most 1 cigarette & $24.3(66)$ & 6.91 & \\
\hline \multicolumn{4}{|l|}{$\begin{array}{l}\text { Belief at baseline that smoking keeps } \\
\text { weight down }\end{array}$} \\
\hline Yes & $20.0(53)$ & 6.94 & 0.4230 \\
\hline No & $80.0(212)$ & 6.59 & \\
\hline \multicolumn{4}{|l|}{ Dieting behaviour } \\
\hline Yes & 29.7180 & 8.21 & 0.0001 \\
\hline No & $70.3(189)$ & 5.99 & \\
\hline \multicolumn{4}{|l|}{ Weight self evaluation } \\
\hline Underweight & $9.4(25)$ & 5.00 & 0.0001 \\
\hline Just about right & $71.8(191)$ & 6.53 & \\
\hline Overweight & $18.8(50)$ & 7.88 & \\
\hline \multicolumn{4}{|l|}{ Depression index } \\
\hline Low & 34.9 (94) & 6.12 & 0.0774 \\
\hline Medium & $33.8(91)$ & 6.86 & \\
\hline High & $31.2(84)$ & 7.03 & \\
\hline \multicolumn{4}{|l|}{ Body mass index $\left(\mathrm{kg} / \mathrm{m}^{2}\right)$} \\
\hline $\mathrm{BMI}<20$ & $58.6(160)$ & 6.47 & 0.4434 \\
\hline $20 \geqslant B M I<23$ & $28.6(78)$ & 6.95 & \\
\hline $23 \geqslant B M I$ & $12.8(35)$ & 6.83 & \\
\hline
\end{tabular}

* Subjects include female adolescents aged 12-15 who had smoked at most 1 cigarette by baseline †Baseline smoking status: non-susceptible non-smoker = girls who had never even puffed and were not susceptible to smoking; susceptible non-smoker = girls who had never even puffed but were susceptible to smoking; smoked at most 1 cigarette $=$ girls who had smoked or puffed at most a whole cigarette.

perceived importance of being thin and belief that smoking keeps weight down by adding an interaction term in the final model. The standard error for the interaction term was calculated using the Wald test. ${ }^{42}$

\section{RESULTS}

\section{Characteristics of the study population}

Table 1 represents the characteristics of the study population, which consisted of female adolescents aged 12-15 years at baseline who reported having smoked at most one cigarette by baseline and who completed the follow up survey $(\mathrm{n}=273)$. A large majority of the subjects were white $(84.1 \%)$, most had engaged in dieting $(70.3 \%)$, and a large majority did not believe that smoking keeps weight down (80\%). Approximately $70 \%$ of the baseline subjects evaluated their weight as just about right. The median BMI $\left(\mathrm{kg} / \mathrm{m}^{2}\right)$ was 19.76 with a range of 11.1-33.9, and the median score on the 10 point personal value of being thin scale was 7 (not shown in the table).

There was no significant variation in perceived importance of being thin by age group, race/ethnicity, education level of the adult informant, baseline smoking status, belief at baseline that smoking keeps weight down, and BMI. Girls who had engaged in dieting were likely to have a higher level of perceived importance of being thin, compared to girls who had not engaged in dieting $\left(T=6.262_{d f} ; p=0.0001\right)$. Weight self evaluation also showed a significant positive association with perceived importance of being thin as girls' evaluation of their weight ranged from underweight to just about right to overweight $\left(\mathrm{F}=9.64_{\text {df } 2} ; \mathrm{p}=0.0001\right)$. In addition, there was a marginally significant increasing trend in perceived importance of being thin with increasing depressive feeling $\left(\mathrm{F}=2.58_{\mathrm{df} 2} ; \mathrm{p}=0.0774\right)$.

Baseline information was compared between study population and excluded subjects. Because of loss to follow up and values for several variables, we excluded 194 subjects who had smoked at most one cigarette by baseline from the original cohort in the primary analysis. We compared the baseline characteristics of study subjects and excluded subjects. There was no significant difference between two groups except baseline smoking status and SES. Excluded subjects were more likely to have experimented with smoking and the adult informants of excluded subjects were less educated.

\section{Predictors of progression to established smoking}

Table 2 shows distribution of baseline characteristics by progression to established smoking by 1997. Twenty three per cent $(n=64)$ of 273 respondents became established smokers during the four year follow up period. The percentage of girls 
Table 2 Baseline characteristics of female adolescents in the study population who had smoked at most one cigarette by baseline by progression to established smoking and adjusted odds ratios

\begin{tabular}{|c|c|c|c|c|}
\hline & \multicolumn{2}{|c|}{$\begin{array}{l}\% \text { progressing to } \\
\text { established smokers† }\end{array}$} & \multirow[b]{2}{*}{$\begin{array}{l}\text { Adjusted } \\
\text { OR‡ }\end{array}$} & \multirow[b]{2}{*}{$95 \% \mathrm{Cl}$} \\
\hline & $\begin{array}{l}\text { Yes 23.4 } \\
(n=64)\end{array}$ & $\begin{array}{l}\text { No } 76.6 \\
(n=209)\end{array}$ & & \\
\hline Perceived value of being thin $(0-10$ scale) & & & 1.15 & 1.019 to 1.293 \\
\hline Low $(0-4)$ & 7.1 & $92.86 * *$ & 1.00 & \\
\hline Medium (5-7) & 23.85 & 76.15 & 3.37 & 1.041 to 10.941 \\
\hline High $(8-10)$ & 29.31 & 70.69 & 4.46 & 1.400 to 16.689 \\
\hline Age (year) & & & 1.32 & 0.978 to 1.777 \\
\hline \multicolumn{5}{|l|}{ Age group } \\
\hline $12-13$ & 19.46 & $80.54^{*}$ & & \\
\hline $14-15$ & 28.46 & 71.54 & & \\
\hline \multicolumn{5}{|l|}{ Race/ethnicity } \\
\hline White, non-Hispanic & 25.44 & 74.56 & 1.00 & \\
\hline Other & 13.95 & 86.05 & 0.57 & 0.212 to 1.507 \\
\hline \multicolumn{5}{|l|}{ Baseline smoking status } \\
\hline Non-susceptible non-smoker & 13.10 & $86.90 * * *$ & 1.00 & \\
\hline Susceptible non-smoker & 18.03 & 81.97 & 1.02 & 0.634 to 2.372 \\
\hline Experimenter & 50.00 & 50.00 & 5.24 & 2.721 to 10.091 \\
\hline \multicolumn{5}{|l|}{ Education level of adult informant } \\
\hline High school or less & 29.17 & $70.83^{* *}$ & & \\
\hline More than high school & 18.67 & 81.33 & & \\
\hline \multicolumn{5}{|l|}{ Belief that smoking keeps weight down } \\
\hline No & 23.11 & 76.89 & & \\
\hline Yes & 26.42 & 73.58 & & \\
\hline \multicolumn{5}{|l|}{ Dieting behaviour } \\
\hline Yes & 23.75 & 76.23 & & \\
\hline No & 23.28 & 76.72 & & \\
\hline \multicolumn{5}{|l|}{ Weight evaluation } \\
\hline Underweight & 20.00 & 80.00 & & \\
\hline Just about right & 25.13 & 74.03 & & \\
\hline Overweight & 20.00 & 80.00 & & \\
\hline \multicolumn{5}{|l|}{ Depression } \\
\hline Low & 18.09 & 81.91 & & \\
\hline Medium & 27.47 & 72.53 & & \\
\hline High & 25.00 & 75.00 & & \\
\hline \multicolumn{5}{|l|}{ Body mass index $\left(\mathrm{kg} / \mathrm{m}^{2}\right)$} \\
\hline $\mathrm{BMI}<20$ & 20.00 & 80.00 & & \\
\hline $20 \geqslant B M I<23$ & 28.21 & 71.79 & & \\
\hline $23 \geqslant \mathrm{BMI}$ & 28.57 & 71.43 & & \\
\hline
\end{tabular}

tEstablished smokers are girls who had smoked more than 100 cigarettes in their life.

$\ddagger$ Multivariate logistic model only include perceived value of being thin, race/ethnicity, age, baseline smoking status, belief that smoking keeps weight down at follow up.

${ }^{*} p<0.1 ;{ }^{* *} p<0.5 ;{ }^{* *} p<0.01$.

who had become established smokers by the follow up time varied significantly by perceived importance of being thin $\left(\chi^{2}=6.1994_{\text {df } 2} ; \mathrm{p}=0.0128\right)$. Girls reported a high value for being thin at baseline were more likely to become established smokers than girls who reported a low value for being thin.

There were no significant associations between becoming an established smoker and age group, race/ethnicity, baseline belief that smoking keeps weight down, dieting behaviour, weight evaluation, depression level, and BMI. However, the proportion of girls becoming established smokers varied by the education level of the adult informant and smoking behaviour reported at baseline. A greater proportion of girls who had adult informants whose education level was high school or less progressed to established smoking compared to girls who had adult informants whose education level was higher than high school $\left(\chi^{2}=4.11_{\mathrm{df}} ; \mathrm{p}=0.043\right)$. Similarly, there was a significant difference in the proportion of girls who progressed to established smoking by smoking status at baseline $\left(\chi^{2}=30.02_{\mathrm{df}} ; \mathrm{p}=0.001\right)$. Females who had smoked at baseline were most likely to have progressed to established smoking at follow up (50\%). Females who had never smoked and were not susceptible to smoking at baseline were less likely to have progressed to established smoking (13.1\%), compared to never smoking females who were susceptible to smoking at baseline (18.0\%).

The final model to predict established smoking at follow up included perceived importance of being thin at baseline, age, race/ethnicity, and baseline smoking status (table 2). Educational level of the adult informant, belief at baseline that smoking keeps weight down, dieting behaviour, weight self evaluation, depression, and BMI did not contribute to the base model separately or in combination $(p>0.2)$. The interaction between perceived importance of being thin and belief at baseline that smoking keeps weight down was further examined by adding the interaction terms between these two variables in the model, but it was not significant $(\mathrm{p}>0.5)$. 
Girls who reported a greater perceived importance of being thin at baseline were significantly more likely to have progressed to established smoking during the four year follow up period, controlling for age, smoking status at baseline, and race/ethnicity $\left(\chi^{2}=5.1840_{\mathrm{df}} ; \mathrm{p}=0.0228\right)$ (not shown in the table). Odds ratios of three levels of perceived importance of being thin on progression to established smoking are shown in table 2. The odds of establishing smoking among girls who valued thinness most strongly were approximately 4.5 times as high as that among girls who valued thinness least strongly $\left(\mathrm{OR}=4.458 ; \chi^{2}=6.3978_{\mathrm{df}} ; \mathrm{p}=0.0114\right)$. In addition, the odds of establishing smoking among girls who valued thinness somewhat strongly were approximately three times as high as that among the girls who valued thinness least strongly $\left(\mathrm{OR}=3.374 ; \chi^{2}=4.1044_{\mathrm{df} 1} ; \mathrm{p}=0.0428\right)$. There was a dose-response relation between perceived importance of being thin and progression to established smoking, which was significant $\left(\chi^{2}=6.0833_{\mathrm{df} 1} ; \mathrm{p}=0.0136\right)$.

\section{DISCUSSION}

To the best of our knowledge, this is the first longitudinal study to examine the effect of perceived importance of being thin on smoking initiation among younger female adolescents. This study assumes weight concerns and dieting behaviour to be intermediate factors in the relation between perceived importance of being thin and smoking initiation. We found that perceived importance of being thin at baseline was a significant predictor of smoking behaviour during a four year period of follow up among female adolescents in Massachusetts. Controlling for age, race/ethnicity, and smoking status at baseline, we found that greater perceived value of thinness was associated with greater likelihood of progressing to established smoking at follow up. Importantly, we found a dose-response relation, with increasing value on thinness associated with increasing risk of smoking initiation. Girls who valued thinness most strongly were approximately four times as likely to have become established smokers as girls who valued thinness least in the population.

There are several important factors to consider in interpreting these data. First, baseline susceptibility to smoking and smoking experience before baseline did not explain the observed relation. We repeated the analysis using the same multiple regression model but with only the subjects who had never smoked at baseline. Perceived importance of being thin was a significant predictor of progression to established smoking. Second, this observed relation was not explained by age. Third, this relation was not explained by other possible confounding factors, such as race/ethnicity, SES, BMI, and depressive symptoms. The odds ratio for perceived importance of being thin did not change after adding these factors to the model. Fourth, the relation was not due to differential loss to follow up by the levels of perceived importance of being thin at baseline. While excluded subjects were more likely to have experimented with smoking and the adult informants of excluded subjects were less educated as a group, there was no significant difference in perceived importance of being thin. Loss to follow up did not vary significantly from lowest perceived importance of being thin to highest. This analysis of loss to follow up suggests that the risk of progression to established smoking may have been somewhat underestimated in the cohort, but does not suggest that the risk estimate for the effect of perceived value of thinness is biased.

Contrary to many previous studies, dieting behaviour at baseline did not predict smoking initiation prospectively in this study. Although weight concerns have repeatedly been found to correlate with smoking status, prospective research has been lacking in this area. Most of the previous studies that found an association between dieting behaviour/weight concerns and smoking were cross-sectional studies. This study is one of the few prospective studies to examine the association and we failed to detect any association. This may suggest that the observed relation is, in part, due to a reverse association.

There are a few possible explanations for the effect found in our study. One possible explanation is that a girl who highly values being thin may have heightened weight concerns that promote dieting behaviour. ${ }^{43}$ Consequently, if she also believes that smoking promotes weight loss, she is likely to develop smoking behaviour, which has been demonstrated by other research. ${ }^{41}{ }^{20}$ Another possible explanation is that perceived importance of being thin influences smoking initiation through psychosocial pathways. A girl who values being slim and has elevated weight concerns is likely to have a negative body image, which is likely to influence psychosocial factors such as self esteem or self worth. ${ }^{12}$ Considering that negative psychosocial states are significant risk factors for smoking initiation among adolescents, ${ }^{511}{ }^{22}$ it is likely that she will have a high risk of smoking initiation. Our study was not able to demonstrate these pathways. Further study is needed to examine the mechanisms of the relation between perceived importance of being thin, weight concerns, and smoking initiation.

Contrary to our hypothesis, there was no effect modification between perceived importance of being thin and belief that smoking keeps weight down. It is not clear in this study why the interaction between perceived importance of being thin and the belief that smoking keeps weight down did not exist. However, one possible reason may be that perceived importance of being thin may predict smoking initiation only partially through weight concerns and, therefore, it may be difficult to detect an interaction effect. Another possible explanation is that belief that smoking keeps weight down may be an accelerator for smoking behaviour only among girls who are interested in smoking. Once girls entered in the process of progression to smoking, the belief may be used for their rationalisation of their smoking behaviour. However, the belief may not affect girls who are not interested in smoking. Camp et al found that belief in smoking as a weight control strategy did not predict regular smoking versus non-smoking but reliably separated experimental smokers from regular smokers. ${ }^{44}$ The authors concluded that belief in smoking as a weight control strategy may be important in moving from experimental to regular smoking but not from never smoking to smoking experimentation. Our data somewhat supported their view. The belief that smoking keeps weight down did not predict smoking initiation prospectively. However, we could not examine the effect of this belief on the transition from experimenter to established smoker due to lack of a sufficient number of experimenters at baseline. A further possibility is that a third variable, such as social pressure from peers, has an effect both on the belief that smoking keeps weight down and the perceived importance of being thin.

As expected, smoking experience at baseline significantly predicted smoking behaviour at follow up; girls who had experienced smoking before baseline were approximately five times as likely to become established smokers at follow up, compared to girls who reported at baseline never having smoked or puffed. We found age to be positively associated with progression to established smoking, which was marginally significant. Race/ethnicity, depression, BMI, and education level of the adult informant were not associated with progression to established smoking at follow up once other factors were controlled.

Our findings suggest that perceived importance of being thin could be a risk factor for smoking initiation as well as for behaviours related to dieting. It has been unclear whether female adolescents initiate smoking with the intention to control weight ${ }^{45}{ }^{46}$ or whether dieting and smoking are both part of a larger constellation of unhealthy behaviours, the onset of which is determined by other variables. ${ }^{11} 1220454748$ 
This research supports the latter view. It seems clear that perceived importance of being thin, before dieting behaviour and smoking behaviour, is crucial for both behaviours. Perceived importance of being thin could be an indicator of social/ environmental factors (for example, an overemphasised value on thinness in a society) that influence adolescent health. ${ }^{49-51}$ A social ideal of appearance for young women in society is considered to influence the perceived importance of being thin among girls, which, in turn, influences their body image and body dissatisfaction, and elevates weight concerns. ${ }^{49-54}$ For example, an overemphasis on the social value of thinness by the mass media may be one plausible social/environmental factor that affects personal value of thinness. ${ }^{5455}$ Further study is needed to examine the mechanisms of this relation.

Many studies have documented that the majority of adolescent girls engage in dieting. ${ }^{12}{ }^{56}$ For example, the 1997 Massachusetts Youth Risk Behavioural Survey showed that $47 \%$ of girls in high school reported dieting in the past 30 days and $63 \%$ of girls reported current weight loss efforts. ${ }^{57}$ Assuming that perceiving the importance of being thin precedes weight loss efforts, the majority of girls in the USA apparently place a high level of importance on being thin. In our population, the majority of girls $(85 \%)$ reported medium or high levels of perceived importance of being thin, and a significant proportion of these early adolescents had already engaged in some dieting behaviours. This means that the majority of early adolescent girls are a high risk population for several risky behaviours, including smoking, which is an alarming implication of the findings of the present study.

This study has several limitations. First, the measurement of the independent variable was based on only one item. It is important to develop a reliable and validated measure of perceived importance of being thin by including more items and testing it psychometrically. However, although the measure we used may be weak psychometrically, the estimated effect of this independent variable was still strong. Therefore, we believe that it will be important to examine the effect of perceived importance of being thin in future studies using validated measures. Second, the majority of our sample comprised white girls, which made it difficult to conduct stratified analyses by race/ethnicity. Because it is plausible that the meaning of thinness varies by race/ethnicity, ${ }^{315859}$ future research should examine the relation between perceived importance of being thin and smoking initiation by race/ ethnicity. ${ }^{11}{ }^{25}$ Finally, we could only include female adolescents because of lack of information on the independent variable among male adolescents. Prior research has shown that there are significant differences by sex in factors associated with smoking initiation and dieting behaviours. ${ }^{11}{ }^{25}$ Without male subjects for comparison, it is difficult to delineate the factors that make females at risk.

Despite these limitations, this study provides significant evidence that perceived importance of being thin may predict smoking initiation among female adolescents over time. The evidence that perceived importance of being thin is a widespread risk factor for smoking initiation as well as other adolescent health problems suggests that it is crucial to broaden the scope of health programmes and policies to protect adolescent health. ${ }^{12} 17202224$ Discussion of the perceived importance of thinness should be included in health promotion programmes for adolescents in order to prevent a wide range of unhealthy behaviours, including smoking. It is also clear that we should pay attention to the social/ environmental factors that make girls feel that thinness is a positive value, and should intervene in the factors that influence perceived importance of being thin in order to improve adolescent health, especially among girls. According to Geoffrey Rose's model, ${ }^{60}$ a population based strategy needs to be considered; it is important to identify factors that decrease the average value placed on thinness by girls. Future studies should attempt to demonstrate the pathways connecting per-

\section{What this paper adds}

Over the past few decades, a great number of cross-sectional studies have established a positive association between weight concerns and cigarette smoking among female adolescents. However, few prospective studies have been conducted to establish a causal relation between weight concerns and tobacco use. To the best of our knowledge, this is the first analysis to examine whether the perceived importance of being thin is an initiating factor for smoking among younger female adolescents.

This prospective study suggests that the level of perceived importance of being thin among young female adolescents predicts future smoking initiation. Smoking prevention programmes designed for female adolescents may therefore benefit from the inclusion of content related to body image and the perceived importance of being thin.

sonal value of thinness and smoking initiation, including factors associated with dieting, as well as investigate the factors that elevate the perceived importance of being thin among girls.

\section{ACKNOWLEDGEMENTS}

This study was supported by the Harvard Tobacco Training Research Grant, which is funded by the Robert Wood Johnson Foundation and by grants from the Robert Wood Johnson Foundation Substance Abuse Policy Research Program (grant 031587) and the Massachusetts Department of Public Health, Massachusetts Tobacco Control Program (Health Protection Fund). We would like to thank Dr Karen Emmons and Dr Lois Biener for comments on an earlier version of this paper.

\section{Authors' affiliations}

K Honjo, Department of Hygiene and Preventive Medicine, Okayama University Graduate School of Medicine and Dentistry, Okayama, Japan M Siegel, Social and Behavioral Sciences Department, Boston University School of Public Health, Boston

\section{REFERENCES}

1 Centers for Disease Control and Prevention. Tobacco use among high school students - United States, 1997. MMWR Morb Mortal Wkly Rep 1998;47:229-33.

2 US Department of Health and Human Services. Tobacco use among U.S. racial/ethnic minority groups: African Americans, American Indians and Alaska Natives, Asian Americans and Pacific Islanders, Hispanics. A report of the Surgeon General, 1998. Atlanta, Georgia: Centers for Disease Control and Prevention, Office on Smoking and Health, 1998 (US Government Printing Office Publication No S/N 017-001-00527-4.)

3 US Department of Health and Human Services. Preventing tobacco use among young people. A report of the Surgeon General, 1994. Atlanta, Georgia: Public Health Service, Centers for Disease Control and Prevention, Office on Smoking and Health, 1994. (US Government Printing Office Publication No S/N 017-001-00491-0.)

4 French SA, Perry CL, Leon GR, et al. Weight concerns, dieting behavior, and smoking initiation among adolescents: a prospective study. Am J Public Health 1994;84:1818-20

5 Klesges RC, Meyers AW, Klesges LM, et al. Smoking, body weight, and their effects on smoking behavior: a comprehensive review of the literature. Psychol Bull 1989;106:204-30.

6 Ogden J, Pauline F. Examination of the use of smoking for weight control in restrained and unrestrained eaters. Int J Eating Disorders 1994; 16:177-85

7 US Department of Health and Human Services. Women and smoking: a report of the Surgeon General 2001. Atlanta: US Department of Health and Human Services, Center for Disease Control and Prevention, Office on Smoking and Health, 2001

8 Gritz ER, Cigarette smoking by adolescent females: Implications for health and behavior. Women and Health 1984;9:103-15.

9 Gritz, ER, Klesges RC, Meyers AW. The smoking and body weight relationship: implications for intervention and post cessation weight control. Ann Behav Med 1989;1 1:144-53.

10 Gritz ER, Nielsen, I, Brooks L. Smoking cessation and gender: the influence of physiological, psychological and behavioral factors. JAMWA 1996:51:35-42.

11 French SA, Jeffery RW. Weight concerns and smoking: a literature review. Ann Behav Med 1995; 17:234-44. 
12 French SA, Story M, Resnick MD, et al. Frequent dieting in adolescents: psychosocial and health behavior correlates. Am J Public Health 1995;85:695-701

13 Charlton A. Smoking and weight control in teenagers. Public Health 1984;98:277-81.

14 Gritz ER, Crane LA. Use of diet pills and amphetamines to lose weight among smoking and nonsmoking high school seniors. Health Psychology $1991 ; 10: 330-5$.

15 Feldman W, Hodgson C, Corber S. Relationship between higher prevalence of smoking and weight concern amongst adolescent girls. Can J Public Health 1985;76:205-6.

16 Halek C, Kerry KH, Crisp AH, et al. Relationship between smoking, weight and attitudes to weight in adolescent schoolgirls. Postgrad Med J 1993;69:100-6.

17 Tomeo CA, Field AE, Berkey CS, et al. Weight concerns, weight control behaviors, and smoking initiation. Pediatrics 1999;104:918-24.

18 Crisp AH, Sedgewich P, Halek C, et al. Why may teenage girls persist in smoking? J Adolesc 1999;22:657-72.

19 Crisp AH, Halek C, Sedgewich P, et al. Smoking and pursuit of thinness in schoolgirls in London and Ottawa. Postgrad Med J 1998:74:473-9.

20 French SA, Perry CL, Leon GR, et al. Changes in psychological variables and health behaviors by dieting status over a three-year period in a cohort of adolescents females. J Adolesc Health 1995;16:438-47.

21 Wright MR. Body image satisfaction in adolescent girls and boys: a longitudinal study. J Youth Adolesc 1989:18:71-83.

22 Fisher M, Schneider M, Pegler C, et al. Eating attitudes, health-risk behaviors, self-esteem, and anxiety among adolescent females in a suburban high school. J Adolesc Health 1991;12:377-84.

23 Frank RE, Serdula MK, Adame D. Weight loss and bulimic eating behavior: changing patterns within a population of young adult women. Southern Med J 1991;84:457-60.

24 Austin SB. Gortmaker SL. Dieting and smoking initiation in early adolescent girls and boys: a prospective study. Am J Public Health 2001;91:446-50.

25 French SA, Perry CL. Smoking among adolescent girls: prevalence and etiology. JAMWA 1996;51:25-8.

26 Pierce JP, Choi WS, Gilpin EA, et al. Validation of susceptibility as a predictor of which adolescents take up smoking in the United States. Health Psychol 1996;15:355-61.

27 Pierce JP, Choi WA, Gilpin EA, et al. Tobacco industry promotion of cigarettes and adolescent smoking. JAMA 1998;279:511-15.

28 Choi WS, Pierce JP, Gilpin EA, et al. Which adolescent experimenters progress to established smoking in the United States? Am J Prev Med 1997; 13:385-91.

29 Pierce JP, Farkas, AJ, Evans $\mathrm{N}$, et al. An improved surveillance measure for adolescent smoking? Tobacco Control 1995;4(suppl 1):S47-56.

30 Tucker LA. Cigarette smoking intentions and obesity among high school males. Psychology Reports 1983;52:530.

31 Killen JD, Taylor CB, Telch M, et al. Depressive symptoms and substance use among adolescent binge eaters and purgers: a defined population study. Am J Public Health 1987;77:1539-41.

32 Patton GC, Hibbert M, Rosier M, et al. Is smoking associated with depression and anxiety in teenagers? Am J Public Health 1996:86:225-230.

33 French AF, Jeffery RW. Consequences of dieting to lose weight: effects on physical and mental health. Health Psychol 1994;13:195-212.

34 Himes JH, Dietz WH. Guidelines for overweight in adolescent preventive services: recommendations form an expert committee. Am J Clin Nutr 1994;59:307-16

35 Radloff LS. The CES-D scale: a self-report depression scale for research in the general population. Appl Psycho Meas 1997;1:385-401.

36 Radloff, LS. The use of the Center for Epidemiologic Studies depression scale in adolescents and young adults. $J$ Youth Adolesc

1991;20:149-66.
37 Biener L, Siegel M. Tobacco marketing and adolescent smoking: more support for a causal inference. Am J Public Health 2000;90:407-10.

38 Pierce JP, Choi WS, Gilpin EA, et al. Validation of susceptibility as predictor of which adolescents take up smoking in the United States. Health Psychol 1996;15:355-61.

39 Choi WS, Pierce JP, Gilpin EA, et al. Which adolescent experimenters progress to established smoking in the United States? Am J Prev Med 1997; 13:385-91.

40 Pierce JP, Farkas AJ, Evans $N$, et al. An improved surveillance measure for adolescent smoking? Tobacco Control 1995;4(suppl 1):S47-56.

41 SAS Institute. SAS/STAT software: changes and enhancements, Release 6.10. Cary, North Carolina: SAS Institute Inc, 1994.

42 Howmer DW, Lemeshow S. Applied logistic regression. New York: John Wiley \&Sons Inc, 1989

43 Field AE, Wolf AM, Herzog DB, et al. The relationship of caloric intake to frequency of dieting among preadolescent and adolescent girls. J Am Child Adolesc Psychiatry 1993;32:1246-52.

44 Camp DE, Kelsges RC, Relyea G. The relationship between body weight concerns and adolescent smoking. Health Psychol 1993;12:24-32.

45 Klesges RC, Mizes JS, Klesges LM. Self-help dieting strategies in college males and females. Int J Eating Disorders 1987;6:409-17.

46 Schwartz DM, Thompson, MG Johnson CL. Anorexia nervosa and bulimia: the socio-cultural context. Int J Eating Disorders 1982;1:20-36.

47 Killen JD, Barr-Taylor C, Telch M, et al. Depressive symptoms and substance use among adolescent binge eaters and purgers: a defined population study. Am J Public Health 1987;77:1539-41.

48 Rovinson TN, Killen JD, Barr-Taylor C, et al. Perspectives on adolescent substance use: a defined population study. JAMA 1987;258:2072-6.

49 Garner DM, Garfinkel PE, Achwartz D, et al. Cultural expectations of thinness in women. Psychological Reports 1980;47:483-91.

50 Silverstein B, Perdue L, Perterson $G$, et al. The role of the mass media in promoting a thin standard of bodily attractiveness for women. Sex Roles 1986;14:519-32.

51 Wiseman CV, Gray JJ, Mosimann JE, et al. Cultural expectations of thinness in women: an update. Int J Eating Disorders 1992;11:85-9.

52 Silverstein B, Perdue L, Peterson B, et al. Possible causes of the thin standard of bodily attractiveness for women. Int J Eating Disorders 1986:5:907-16.

53 Ogden J. Fat chance! The myth of dieting explained. UK/USA: Routledge, 1992

54 Tiggemann M, Pickering AS. Role of television in adolescent women's body dissatisfaction and drive for thinness. Int J Eating Disorders 1996;20:199-203.

55 Field AE, Camargo CA, Taylor CB, et al. Relation of peer and media influences to the development of purging behaviors among preadolescent and adolescent girls. Arch Pediatr Adolesc Med 1999;153:1 184-9.

56 American School Health Association for the Advancement of Health Education, Society for Public Health Education, Inc. The national adolescent student health survey: a report on the health of America's youth. Oakland, California: Third Party Publishing Co, 1989.

57 Massachusetts Department of Education. 1997 Massachusetts Youth Risk Behavioral Survey Results. Malden, Massachusetts: Massachusetts Department of Education, 1998.

58 Dawson DA. Ethnic differences in female overweight: data from the 1985 National Health Interview Survey. Am J Public Health 1988;78:1326-9

59 Kelm MC, Klesges RC, Bene CR, et al. A psychometric study of restraint: the impact of race gender weight and marital status. Addict Behav 1990;15:147-52.

60 Rose G. The strategy of preventive medicine. New York: Oxford University Press, 1992. 